Proceedings

\title{
Small Displacement Measurement of Crack Opening Using One Pitch Phase Analysis (OPPA) Method ${ }^{+}$
}

\author{
Yoshiharu Morimoto *, Akihiro Masaya, Thomas Yata, Duy Anh Luong and Quentin Quarles \\ 4D Sensor Inc., 649-3-111, Naka, Wakayama 640-8451, Japan; masaya@4d-sensor.com (A.M.); \\ yata@4d-sesnor.com (T.Y.); duy@4d-sensor.com (D.A.L.); quentin@4d-sensor.com (Q.Q.) \\ * Correspondence: morimoto@4d-sensor.com; Tel.: +81-73-454-1004 \\ + Presented at the 18th International Conference on Experimental Mechanics (ICEM18), Brussels, Belgium, \\ 1-5 July 2018.
}

Published: 29 June 2018

Abstract: A new method to measure small displacement of crack width.

Keywords: displacement measurement; crack opening displacement; one-pitch phase analysis; sampling moiré method; phase analysis; grating

\section{Introduction}

Infrastructures such as bridges and building are deteriorating, and inspection is becoming important. When a crack occurs in a concrete bridge, steel bridge, or a building, it is important to perform a safety check to confirm whether the crack width increased or decreased. Currently, the crack width is measured by human eye or by a $\pi$ gauge with a strain gauge [1]. The resolution of the method by human eye is not suitable due the amount of time it takes and it is quite dangerous to work at high altitudes on the infrastructures. The $\pi$ gauge is very expensive and is not useable for bridges due the required amplifier and power supply. Although measuring methods using Moiré etc. have also been proposed, these systems are not easy to use and the accuracy is not high [2,3].

On the other hand, the authors previously proposed sampling moiré method [4] and one-pitch phase analysis (OPPA) method [5]. In the sampling moiré method, phase shifted moiré fringes are generated by down-sampling (thinning-out) of a grating image, and deformation of the grating is measured accurately by the phase analysis. In the OPPA method for measuring the shape of an object, the phase of a grating projected onto the object is detected by using the brightness data of one pitch of the grating, and then the 3D shape is analyzed with higher precision. In this research, the authors propose a method to measure small displacements of objects such as cracks using the sampling moiré method and the OPPA method. It is possible to measure incremental displacement of a crack width precisely and inexpensively. The theory and the applications are shown.

\section{Phase Analysis of Grating}

To analyze shape or deformation, grating phase analysis methods such as phase shifting method [6] and Fourier transform profilometry [7] are used. These phase analysis of grating provides accurate 3D shape or deformation.

Figure 1 shows the schematic grating images obtained by shifting the phase of projected grating onto an object for measuring 3D shape of the object. In Figure 1a, phase shifted nine images are shown. The brightness distributions along the center lines of the images are shown in Figure $1 \mathrm{~b}$. The brightness change during one cycle of phase shifting at the end of the center lines is shown with red points. The Phase shifting method uses these brightness data of red points in Figure 1a,b. However, it is also possible to analyze the phase by using blue points for one cycle along the center line of a single-shot 
image. Sampling moiré method and OPPA method use these blue point data for analyzing the phase. In phase shifting method, the object should not move during phase shifting. On the other hand, sampling moiré method and OPPA method are useful for real-time or high-speed measurement for moving objects because the phase analysis can be performed from a single-shot image of a structure with a grating.

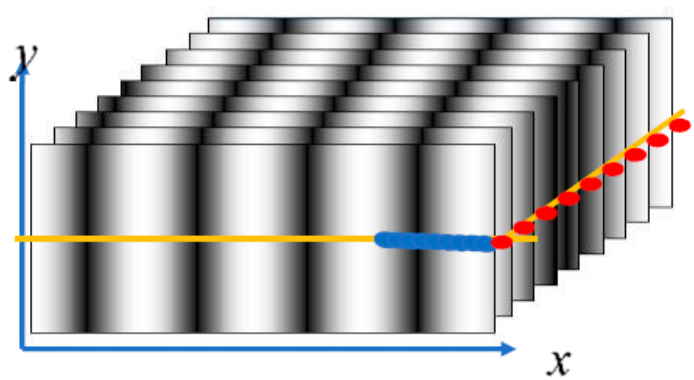

(a)

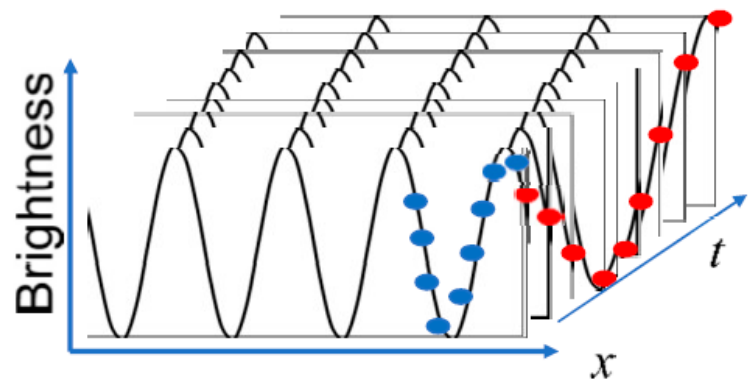

(b)

Figure 1. Explanation of phase analysis for phase shifting method and OPPAmethod. (a) Phase shifted grating images; (b) Brightness distributions along center line.

\subsection{Sampling Moiré Method [4]}

The theory of the sampling moiré method is shown in Figure 2. Figure 2a shows pixel points which are sampling points of a camera. Figure $2 b$ is the grating on an object. Figure $2 c$ is the images taken at the pixels. Figure $2 \mathrm{~d}-\mathrm{g}$ show sampled image by changing the thinning-out phase that is, down sampling. Figure $2 \mathrm{~h}-\mathrm{k}$ show interpolated images from Figure $2 \mathrm{~d}-\mathrm{g}$. They show phase- shifted moiré fringes. From these images the phase can be analyzed by phase shifting method.

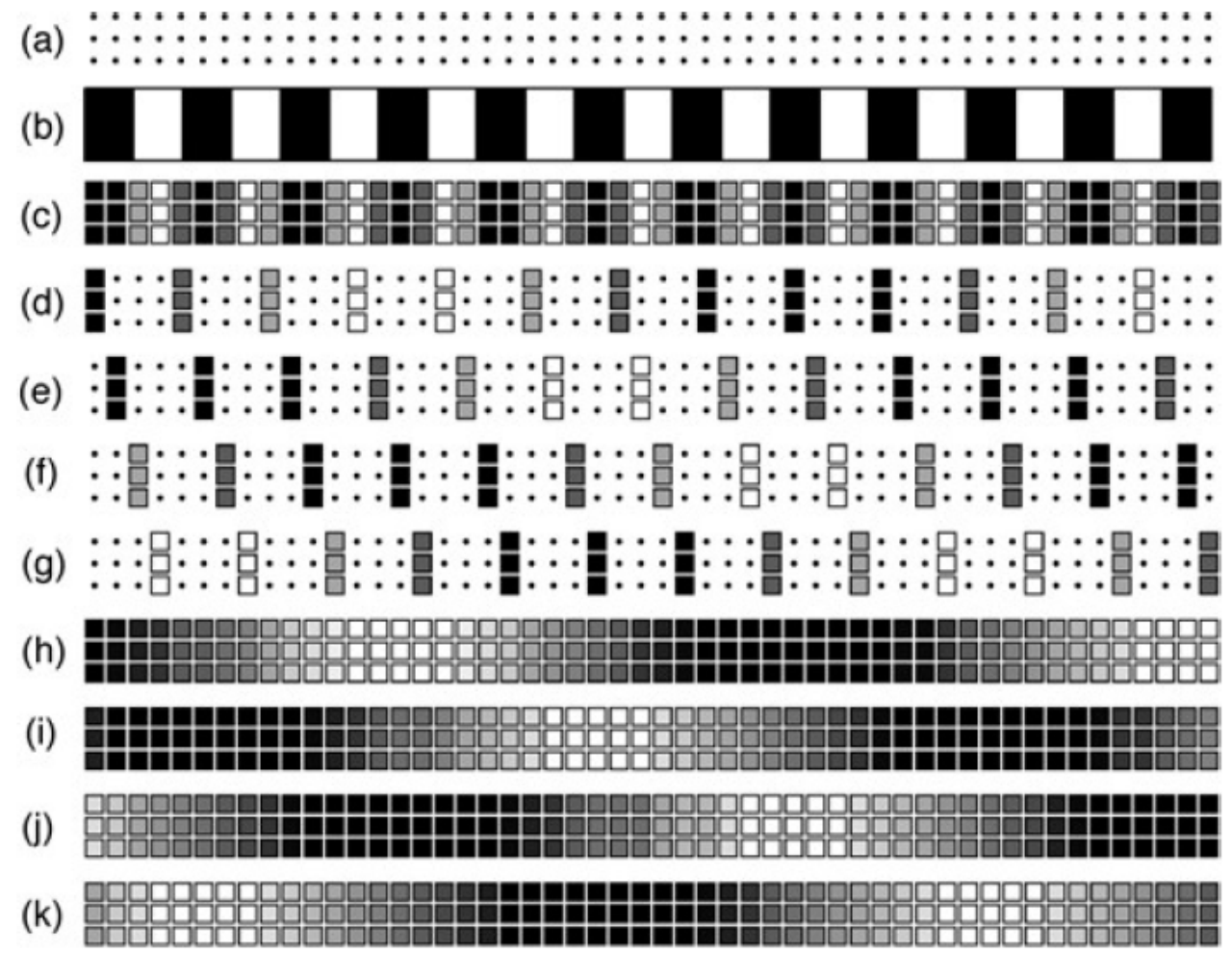

Figure 2. Schematic explanation of sampling moiré method and example applied to rubber plate with holes. 


\subsection{One-Pitch Phase Analysis (OPPA) Method [5]}

The authors have previously proposed a new method to analyze the phase of a grating using brightness data of one pitch of the grating without interpolation shown in Sampling Moiré Method.

To measure 3D shape, a moiré topography optical system is used as shown in Figure 3. If the pixel pitch is adjusted to $N$ pixels of the camera for one-pitch of the grating image, the pixel number is constant $N$ at any height of the object and only the phase of the one-pitch grating changes according to the height. The system does not require the control system for projection of phase-shifting.

The accuracy is fine, and the analysis speed is higher than the 3D shape measurement methods using sampling moiré method. The cost of the system is very low. This method was applied to modal analysis of a rubber plate under changing vibrating frequency. In this study, the OPPA method was applied to analyze the phase of the continuous brightness data of one pitch of the grating.

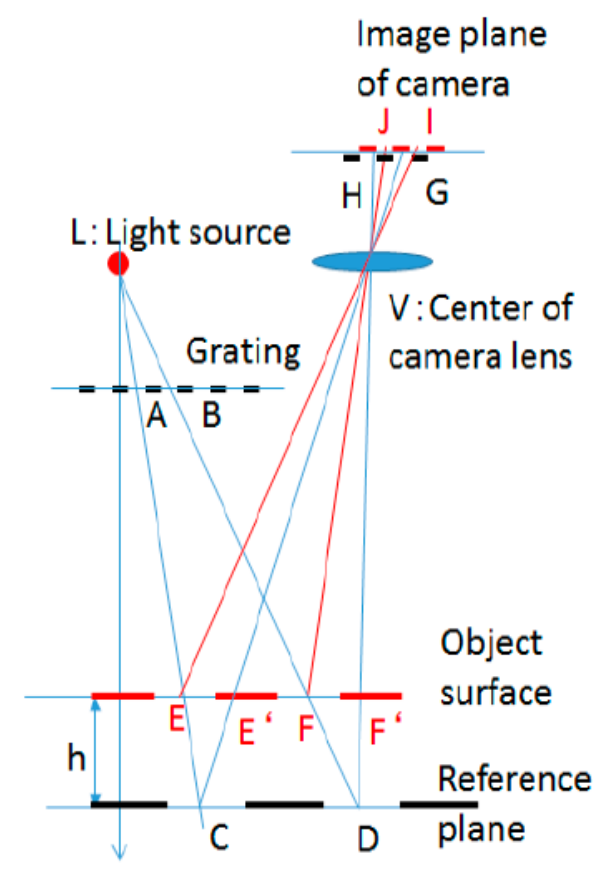

Figure 3. Schematic optics of OPPA method.

\subsection{Homography Transformation}

To perform accurate phase analysis of the grating, it is necessary to take a photograph so that the one pitch of the grating matches an integer number of pixels strictly. To do this, the grating photograph should be taken from the front in the direction perpendicular to the grating plane; this is troublesome for users. Even when the camera is facing an arbitrary direction, if the grating image is converted so that the image is viewed from the front and it has an integer number of pixels for one pitch of the grating by homography transformation, photographing will be easier. In this paper, the effects and the accuracy are examined by the homography transformation.

\section{Grating for Crack Measurement}

A set of three gratings used in this study is shown in Figure 4. These gratings are made of 0.3 $\mathrm{mm}$ thickness stainless steel plate. It is composed of aligned $0.5 \mathrm{~mm}$ black squares with $1 \mathrm{~mm}$ pitch in the $x$ and $y$ directions, respectively. The rectangular grating plates $\mathrm{A}$ and $\mathrm{B}$ are pasted on both sides of the crack which is along the y-direction as shown in Figure 4a.

The deformation of the grating changes the phase. However, the camera angle changes the deformation of the grating. To cancel the effect of the phase deformation according to the camera angle, gratings C, D, E and F are set on the same outer grating plate as shown in Figure $4 \mathrm{~b}$. They do not deform because the outer grating plate is pasted at only the right edge far from gratings $\mathrm{C}, \mathrm{D}, \mathrm{E}$ 
and F. Gratings $\mathrm{C}$ and $\mathrm{D}$ are the same $x$-directional position and apart the same $y$-directional distance from the grating A. Gratings $\mathrm{E}$ and $\mathrm{F}$ are the same $x$-directional position apart the same distance from the grating B. At the outer 4 corners, there are 4 markers for homography transformation. By detecting these markers, it is possible to automatically analyze the grating positions and the displacements.

Near the crack tip of a structure, the distributions of the displacement and the strain change greatly. However, at deviated positions from the crack tip, most of the displacement occurs at the cracked part, and at the other portions other than the crack. Thus, the deformation is almost rigid body deformation or substantially uniform elastic deformation. These gratings are photographed and the average displacements within each region of the gratings are measured by the sampling moiré method and/or the OPPA method.

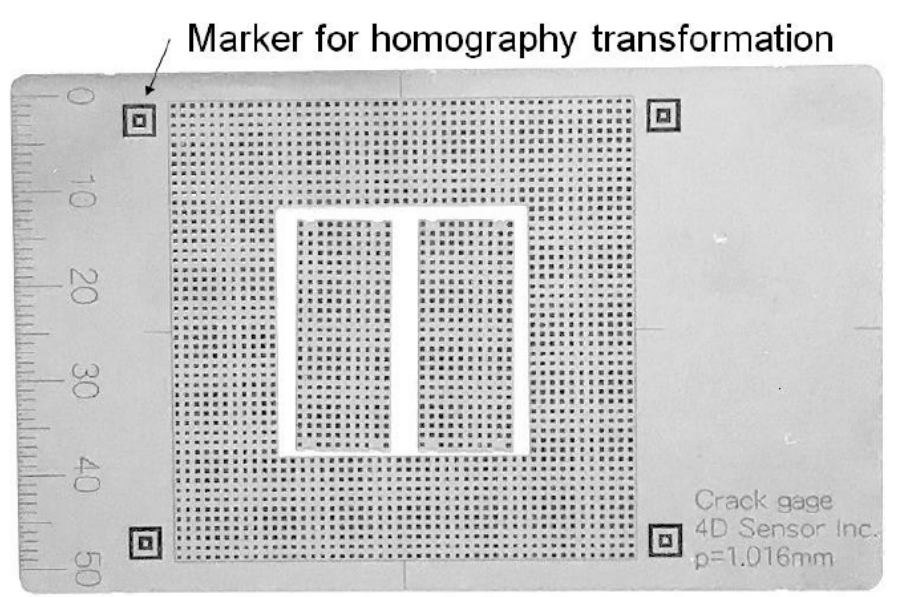

(a)

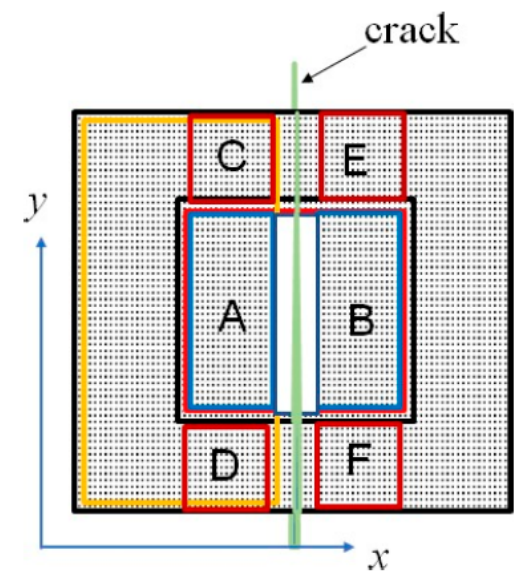

(b)

Figure 4. Grating for crack displacement measurement. (a) Photograph; (b) Schematic image of grating.

To obtain the displacement, the phase of each plate is analyzed from continuous brightness data of one pitch of the grating. The brightness data is Fourier transformed and frequency one is extracted from the frequency spectrum. The inverse Fourier transform provides a very natural cosinusoidal wave and the phase calculation provides very accurate phase information. After calculating the phase difference between the phases before and after deformation, the phase differences in both the rectangular areas on both sides of the crack are averaged. The phase difference provides the increment of the crack opening width.

However, in photography, the position and direction of the camera are different each time. Even in this case, if each image is converted to a view seen from the front using the Homography transformation and one pitch can be accurately matched to an integer pixel, the phase analysis of the grating is performed with high accuracy.

The increment $\Delta u$ of crack width is obtained from the average phases $\emptyset_{B}, \emptyset_{A}, \emptyset_{E}, \emptyset_{C}, \emptyset_{F}$ and $\emptyset_{D}$ in the regions $A, B, C, D, E$ and $F$, respectively, using Equation (1).

$$
\Delta u=\frac{\left(2 \varnothing_{B}-2 \varnothing_{A}-2 \varnothing_{E}-2 \varnothing_{C}-2 \varnothing_{F}-2 \varnothing_{D}\right)}{4 \pi} p
$$

\section{Experimental Set-Up}

Figure 5 shows the experimental set-up used in this paper. A precision moving table which moves vertically on a vibration isolation table is used for providing accurate displacement. The resolution of this moving table is $0.1 \mu \mathrm{m}$. The gratings shown in Figure 4 are used by rotating 90 degrees shown in Figure 5. The upper grating plate $\mathrm{A}$ is attached to the moving part of this moving table. The lower grating plate B is attached to the jig placed on the vibration isolation table. At the same time, 
the lower end of the peripheral grating plates with the regions $C, D, E$ and $F$ is fixed to the jig. The displacement of the grating plate is given by moving the precision moving table upward. The grating is recorded by a camera (Omron Sentech STC-MBS241U3V) with $1920 \times 1200$ pixels from the normal direction to the grating plates. In Section 5.2, the grating is recorded from oblique directions at an angle of $0^{\circ}, 5^{\circ}, 10^{\circ}, 15^{\circ}, 30^{\circ}, 45^{\circ}$ to the normal direction of the grating plate.

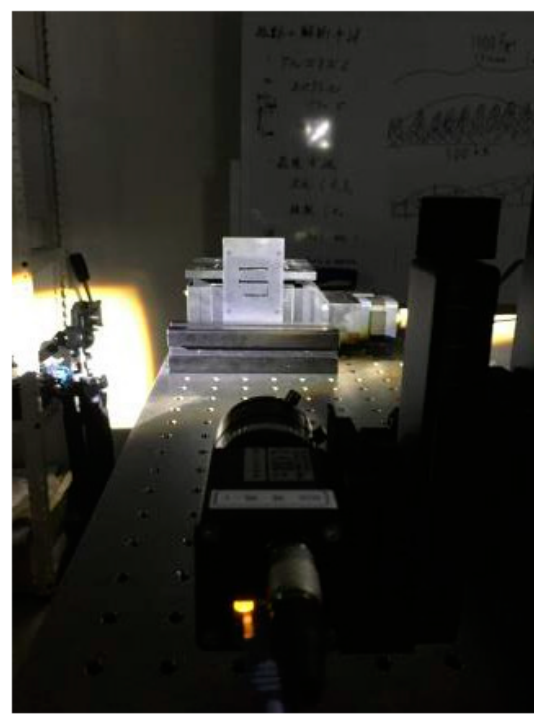

(a)



(b)

Figure 5. Experimental set-up. (a) Front view of set-up; (b) Bird eye view of moving table and grating.

\section{Experimental Results}

\subsection{Measurement Error When Photographing from the Front}

Figure 6 shows the error between the amount of movement given to the moving table and the amount of the displacement analyzed by the sampling moiré method and the OPPA method when the gratings are photographed from the front apart from the grating. The error is the difference between the given displacement of the moving table and the analyzed displacement amount. As a result, the accuracy is within the range of 0 to $2.3 \mu \mathrm{m}$. The displacement increment can be measured very accurately.

There is not a remarkable difference in the errors analyzed by the Sampling moiré method and the OPPA method.

\subsection{Measurement Error When Photographing from Oblique Direction}

Figure 7 shows the displacement errors when the camera optical axis from the axis perpendicular to the grating plane is changed to be 30 degrees. From this, the errors are almost same as Figure 6 . The displacement can be measured with extremely high accuracy even when it is photographed from an oblique angle. 


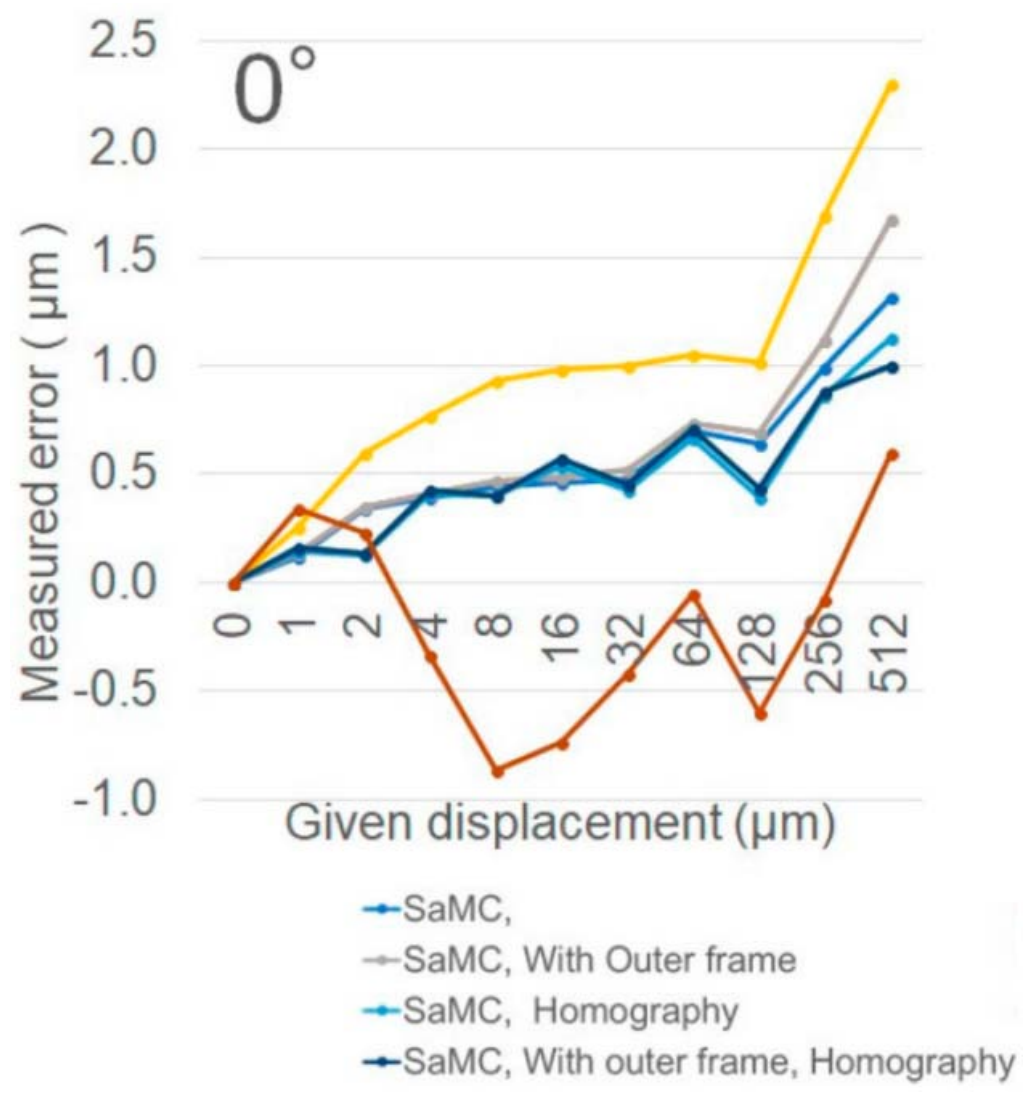

Figure 6. Errors ofdisplacement increment when recoded from the front.

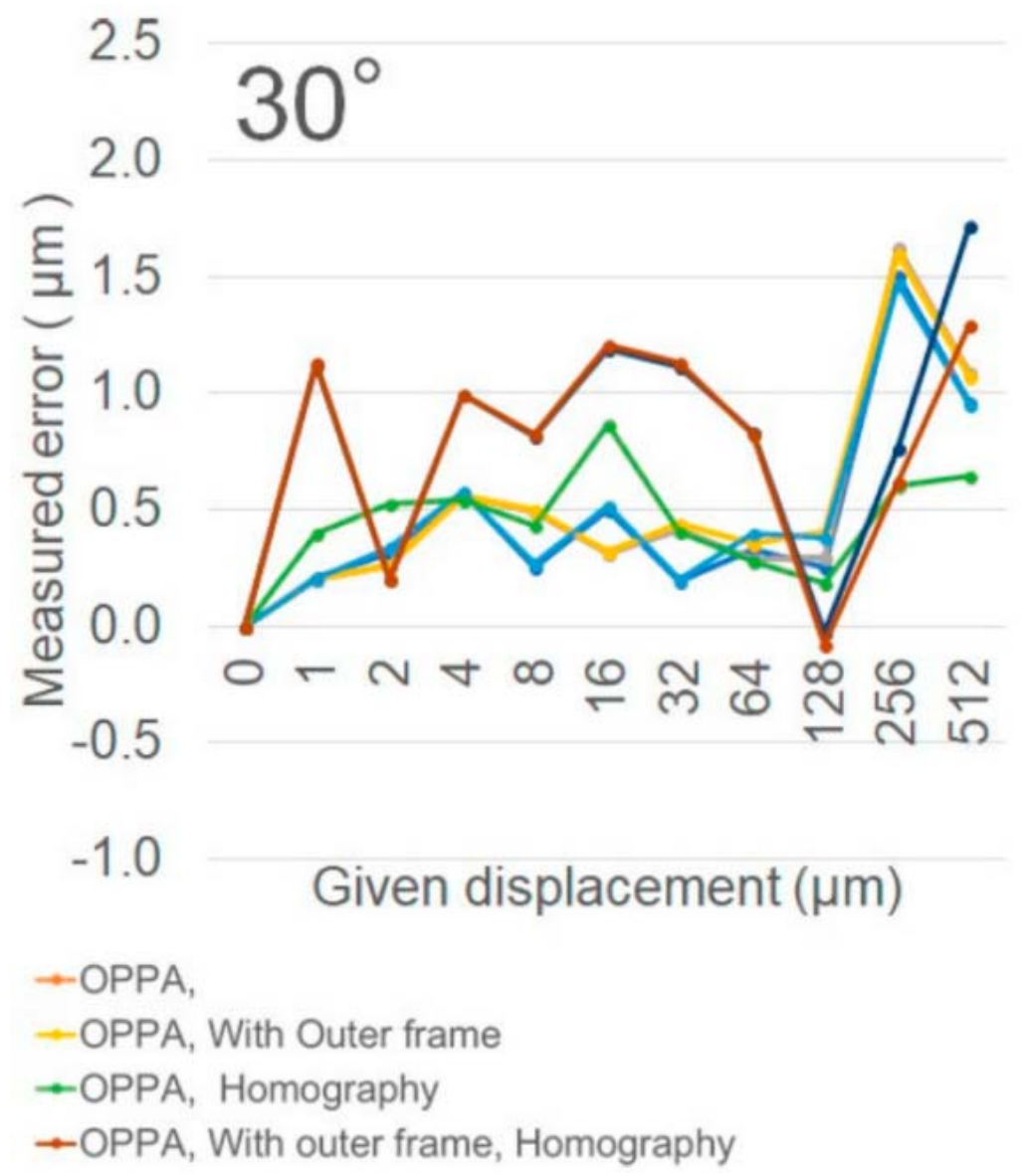

Figure 7. Errors of displacement increment when recorded from 30-degrees angled camera. 


\subsection{Measurement Errors in the Cases with and without Homography Transformation}

In the case of the experiments in Figures 6 and 7, phase analysis is also performed with and without the homography transformation. The results also contain the same order errors with good accuracy.

\subsection{Repeatability}

Experiments in Section 5.1 were repeated 100 times. The standard deviations of the respective errors are examined for the cases with and without the outer grating frame, and the cases with and without homography transformation. The standard deviations of all the errors in each case are shown in Table 1. Each standard deviation of the accuracy in the four case is very small, and the accuracy is approximately $0.2 \mu \mathrm{m}$.

Table 1. Standard deviations for changing conditions.

\begin{tabular}{cccc}
\hline & & With Homography & Without Homography \\
\hline \multirow{2}{*}{ Sampling moiré method } & With outer frame & 0.14 & 0.13 \\
& Without outer frame & 0.2 & 0.13 \\
\cline { 2 - 4 } OPPA method & With outer frame & 0.14 & 0.15 \\
& Without outer frame & 0.21 & 0.15 \\
\hline
\end{tabular}

\subsection{Accuracy When Rotating Camera}

When the camera is rotated around the axis perpendicular to the grating plane, the accuracy is examined. The photos are shown in Figure 8. The displacement differences between the data in Case 1 and the other cases are analyzed. As shown in Figure 8, when the camera rotates, it becomes slightly larger with an error of $-2 \mu \mathrm{m}$.

\begin{tabular}{c|c|c}
\hline \multicolumn{2}{c}{} & Unit $(\mu \mathrm{m})$ \\
\hline \multirow{2}{*}{ Sampling moire method } & Standard deviation \\
\cline { 2 - 3 } OPPA method & Witout outer frame & 0.92 \\
\hline \multirow{2}{*}{ OPA } & 1.94 \\
\cline { 2 - 3 } & With outer flame & 1.86 \\
\cline { 2 - 3 } & Witout outer frame & 2.04
\end{tabular}
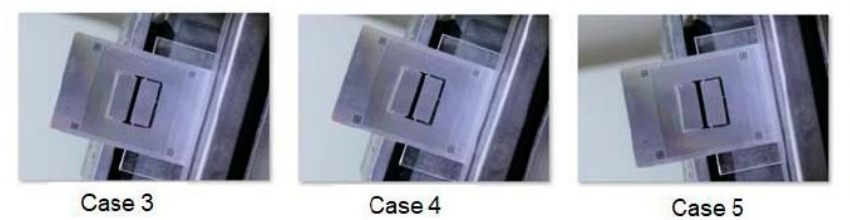

Case 5

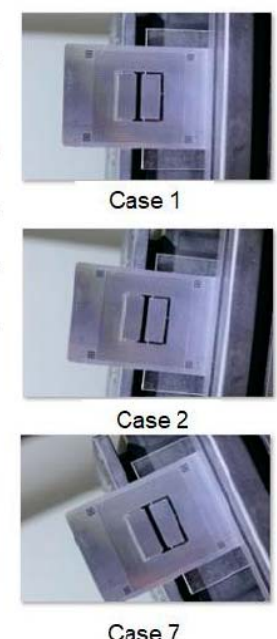

Case 7

Figure 8. Standard deviations analyzed using homography transformation when rotating camera.

\section{Conclusions}

The proposed methods using the sampling moiré method and the OPPA method are useful in measuring the increment of crack width. The accuracy is almost $1 \mu \mathrm{m}$. When conditions are good, crack width increments can be measured with a resolution of $0.1 \mu \mathrm{m}$. The proposed methods are very robust. In this study, the data number is not so large, and a few conditions are examined. In the near future, many experiments under various conditions will be examined

Up to now, bridges have not been periodically inspected. Recently, bridges in Japan are inspected once every five years. However, after a crack has occurred, it is dangerous to wait five years for another inspection. It is necessary to inspect as frequently as possible and constantly monitor 
whether the crack is increasing. By using the proposed method, it is possible to detect the increment by inspecting every other day so that an early warning can be issued.

\section{References}

1. Strain

$$
\text { Gauge }
$$

Type

Transducer

2017.

Available

online: http://www.tml.jp/e/product/transducers/catalog_pdf/PI.pdf (accessed on 4 May 2018).

2. Horiuchi, H. Development of Displacement Measurement System Using Moiré (Measurement Principle and Image Analysis). In Proceedings of the 47th Geotechnical Conference on Japan Geotechnical Society, Hachinohe, Japan, Available online: http://www.ysc-aqua.co.jp/doc/presen_2012jgs.pdf (accessed on 6 May 2018). (In Japanese)

3. Reading-Moiré Tell Tales. Available online: http://www.moiretelltales.com/ (accessed on 15 March 2017).

4. Ri, S.; Fujigaki, M.; Morimoto, Y. Sampling Moiré Method for Accurate Small Deformation Distribution Measurement. Exp. Mech. 2010, 50, 501-508.

5. Morimoto, Y.; Kusunoki, Y.; Ueki, M.; Takagi, A.; Masaya, A. Dynamic Deformation Measurement by OnePitch Phase Analysis (OPPA) Method. In Proceedings of the 11th ISEM'16, Ho Chi Minh, Vietnam, 2-4 November 2016.

6. Bruning, J.H.; Herriott, D.R.; Gallagher, J.E.; Rosenfeld, D.P.; White, A.D.; Brangaccio, D.J. Digital wave front measuring interferometer for testing optical surface and lenses. Appl. Opt. 1974, 13, 2693-2703.

7. Takeda, M.; Ina, H.; Kobayashi, S. Fourier-transform method of fringe-pattern analysis for computer-based topography and interferometry. J. OSA 1982, 72, 156-160.

(C) 2018 by the authors. Licensee MDPI, Basel, Switzerland. This article is an open access article distributed under the terms and conditions of the Creative Commons Attribution (CC BY) license (http://creativecommons.org/licenses/by/4.0/). 